


\title{
Molecular Microstructure in Thermotropic Liquid Crystals Studied by Nuclear Quadrupole Resonance Spectroscopy
}

\author{
Daniel J. Pusiol and Esteban Anoardo \\ Facultad de Matemática, Astronomía y Física, \\ Universidad Nacional de Córdoba, \\ Ciudad Universitaria, 5000 Córdoba, Argentina
}

Received 29 October, 1998

\begin{abstract}
Nuclear Quadrupole Resonance studies in Nematic and Smectic C thermotropic liquid crystalline mesophases of p-heptyl azoxyanizole are reported. The local molecular microstructure is modeled from NQR spectra. In the Smectic C mesophase, the NQR data is explained by assuming two features: i) biaxiality in the electric field gradients at the sites occupied by ${ }^{14} \mathrm{~N}$ nuclei, and ii) the elemental unit cell of the mesophase is composed by two HpAB molecules. These bimolecular units coexist together with single molecules and the relative proportions change with temperature, the quantity of individual molecules being predominant as the temperature approaches the Nematic phase transition. At the lowest temperatures in the Nematic mesophase the NQR spectrum behaves similarly to the corresponding at low temperatures in the $\mathrm{SmC}$ mesophase; this means that we can propose again the existence of a huge number of bimolecular and biaxial unit cells. At higher temperatures the transition to single molecular units is deduced from NQR spectra. That transition is characterized by the one describing the passage from a partially disordered molecular system -the system is conserving some local anisotropic properties of symmetry from the crystalline solid state- to a partially ordered system that resembles an oriented liquid.
\end{abstract}

\section{Introduction}

From the discovery of liquid crystals (LCs) one hundred and ten years ago, a lot of progress has been achieved in the synthesis and in characterization of macroscopic properties. In addition, we can observe very interesting and diverse applications in high technological consuming devices. However, few groups are investigating microscopic molecular arrangements and dynamics, specially in the slow motions time scale. As it is an important physical tool, in this paper we summarize recent advances in understanding molecular local ordering in nematic $(\mathrm{N})$ and smectic $(\mathrm{Sm})$ liquid crystalline mesophases, which can be used to design technologically applicable materials.

The experimental technique we select for our present study is the Nuclear Quadrupole Resonance, NQR. From last years NQR has been established to be a powerful tool to study local molecular order in crystalline as well as in partially disordered solids [15]. power of the technique has been extensively tested in many studies as, for instance, weak distortions and slow dynamical pretransitional effects near a second order phase transitions [6], the determination of thermally activated vacancies in molecular solids [7], etc.

From the first NQR experiments in liquid crystals, it has been established the utility of the technique to study local molecular ordering properties $[8,9]$. The main advantage is due to the easy identification of NQR nuclei spectra in comparison with the always complex proton Nuclear Magnetic Resonance NMR spectral information. Conventional NQR experiments in LCmesophases have been up to now unsuccessful, mainly by two facts:

1. the relative low abundance of quadrupole nuclei, 
even in simplest low molecular weight LCs; and,

2. the relatively strong molecular fluctuations average of the electric field gradients EFGs at the observed NQR-active nuclear sites.

However, NQR spectra have been measured via two similar proton Zeeman-quadrupole cross relaxation techniques; i.e.,

1. crossover relaxation in the laboratory frame at a fixed relaxation period COR [8]; and,

2. from quadrupole dips (QDs), observed in the spinlattice relaxometry profiles $[10,11,12]$.

In both techniques the Zeeman magnetic field is scanned over enough range, in order to overlap both quadrupole and Zeeman spin systems. Consequently, the NQR spectra reconstructed QDs as well as COR minima are distorted by the Zeeman field. In solids, unperturbed NQR spectra can be alternatively recorded from zero-magnetic field Nuclear Quadrupole Double Resonance NQDOR technique [13]. The experiments involve both Field Cycling NMR of protons -from high to zero magnetic field-, together with the irradiation of the quadrupole ${ }^{14} N$ nuclei by means of a second radiofrequency, just during the period of zero Zeeman magnetic field.

In order to describe the advances in the study of molecular microstructures by NQR, we organize this paper as follows:

1. An introduction to NQR technique fundaments is described in the next section making reference to spin $I=1$ quadrupole spectra predicted for:
(a) crystalline solids,
(b) partially disordered solids,
(c) isotropic liquids, and
(d) oriented liquids.

2. Some previous experimental results, the QDs experimental set-up together with some recent improvements are shown in the third section.

3. Experimental measurements carried out in the smectic C (SmC) mesophase of p-heptyl azoxybenzene $(\mathrm{HpAB})$ are analyzed assuming a local molecular self-organization whose basic cell is bimolecular.
4. In the fifth section a brief reference to the NQDOR technique and its experimental set-up is given.

5. The experimental behaviors of NQDOR spectra at several temperatures in both the $\mathrm{SmC}$ and nematic $\mathrm{N}$ mesophases of $\mathrm{HpAB}$ are described in the following section. The compatibility of NQDOR spectral data with those from the previous QDs experiments is analyzed in SmC-HpAB. At low temperatures in the N-phase NQDOR spectra can be interpreted by proposing local EFG biaxiality at the ${ }^{14} \mathrm{~N}$ nuclei sites. At higher temperatures, always within the $\mathrm{N}$-phase range, the transition to a uniaxial EFG symmetry can be deduced from our NQDOR experiments.

6. A brief summary is added at the last section.

\section{NQR fundamentals and spec- tra}

The first non-zero anisotropic expansion term of the electric energy is used to describe the interaction between two electric charge distributions: one the nuclear and the other composed by all the electric charges outside the volume occupied by the quadrupolar nucleus. In fact, for our purposes we can reduce ourself to represent that nuclear interactions through the interaction energy of the nuclear quadrupole moment tensor $\mathcal{Q}$ and the electric field gradient tensor $\mathcal{V}$ valuated at the site occupied by the resonant quadrupole nucleus. Therefore, the NQR Hamiltonian $\mathcal{H}_{\mathcal{Q}}$ is the product $\mathcal{Q}$ and $\mathcal{V}$ tensors. The NQR spectrum for a single $I=1$ nucleus (for instance, ${ }^{14} \mathrm{~N}$ or deuterium ${ }^{2} H$ ) is constituted by three lines: a high frequency doublet -for ${ }^{14} N$ it is located generally in the megahertz frequency scale-, and a single resonance situated in the $\mathrm{kHz}$ 's for the same nucleus. If the $\mathrm{z}$-direction of the principal reference system is defined in the direction of the maximum component of $\mathcal{V}\left(V_{z z}=q\right)$, the three resonance lines are related with the quadrupole coupling constant $\kappa$ and the asymmetry parameter $\eta$ by the expressions:

$$
\nu_{ \pm}=\kappa[1 \pm(1 / 3) \eta]
$$




$$
\begin{gathered}
\nu_{o}=(1 / 3) \kappa \eta, \\
\kappa=\frac{e^{2} q Q}{\hbar}
\end{gathered}
$$

and

$$
\eta=\frac{\left(V_{x x}-V_{y y}\right)}{V_{z z}}
$$

where $e$ is the electron charge, $Q$ is the quadrupole moment of the nucleus and $\hbar$ is the Planck's constant.

Note that $\eta$ describe the departure of $\mathcal{V}$ from the cylindrical geometry and it is, in consequence a measure of the EFG biaxiality. We should to point out here too that the quantization axis is defined by the direction of $V_{z z}$; therefore, the biaxiality is also referred to the two axes which are perpendiculars to that direction. That reference system is mounted on the studied molecule.

As it is shown in Fig. 1, HpAB molecule possesses two ${ }^{14} N$ nuclei. The two nuclei could be unequivalent, or in other words they sense different $\kappa$ 's and $\eta$ 's at its respective positions.

In the case of a crystalline solid with a molecular structure like the one described in Fig. 1, we can expect in general a NQR spectrum composed by six quadrupole lines, see Fig. 2a and refs. [10, 11, 1, 14]. Even more, there are a large quantity of solids with unit cells constructed with two unequivalent molecules. A complex twelve resonances spectrum (three lines for each of the unequivalent ${ }^{14} \mathrm{~N}$ is predicted for such a case. Coming back to the unimolecular unit cell example, but introducing some local disorder we can expect a broadening of the NQR lines produced by local dispersion of both $\kappa$ and $\eta$; see Fig. 2b. In the case of a spherically symmetric EFG, where the spacial anisotropy of $\mathcal{V}$ disappears by the random molecular averaging, the NQR lines completely disappears, see Fig. 2c; i.e., the case of a isotropic liquid. Finally we will consider the case of an oriented liquid; as it is a "rod-like" nematic liquid crystal. There are in this case fast rotations of the LC molecule following the long axis, averaging the EFG asymmetry.. In consequence, the EFG at the quadrupole nucleus position is being uniaxial. See in Fig. 2d the expected NQR spectrum schematically drawled.

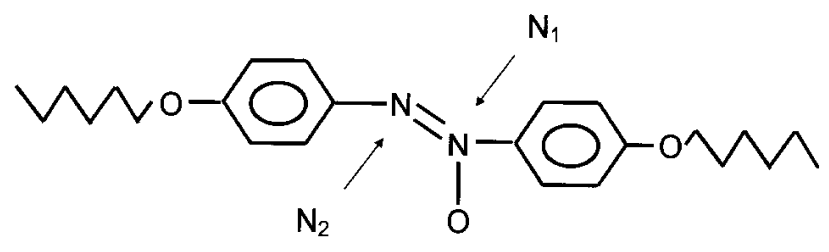

Figure 1. The $H p A B$ molecular structure. Nitrogens $N_{1}$ and $N_{2}$ sense in general each one different values of $\kappa$ and $\eta: \kappa_{1}, \kappa_{2}, \eta_{1}$ and $\eta_{2}$.

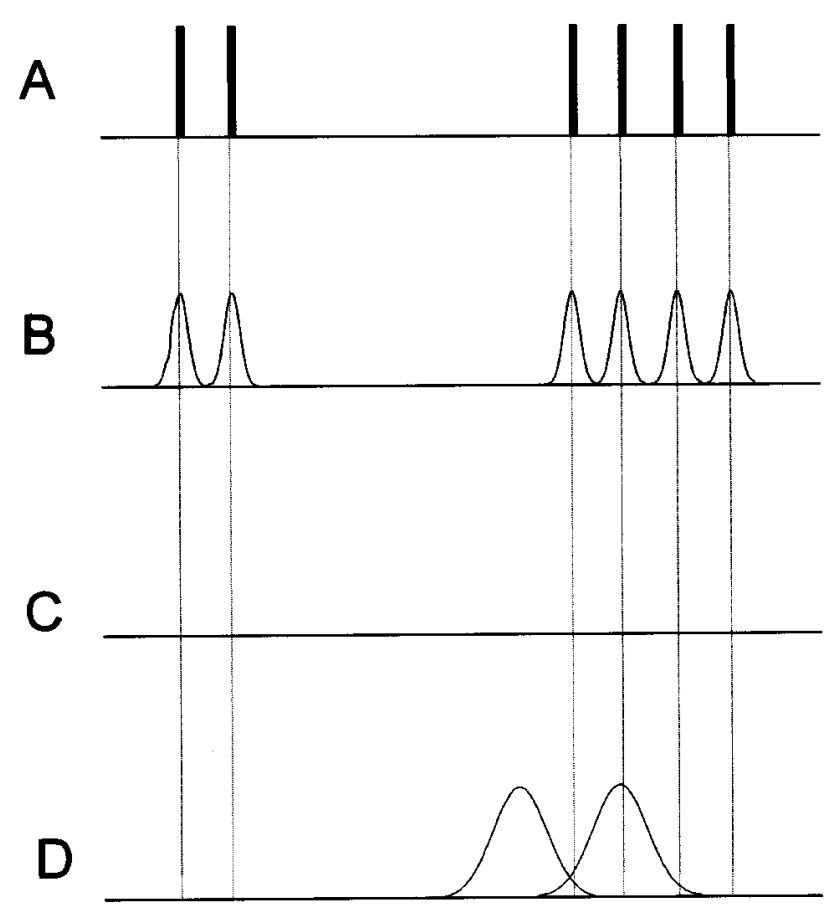

Figure 2. Schematic representation of the NQR spectrum expected for a molecule like the $H p A B$-with two unequivalent ${ }^{14} N$ nuclei, in the following situations: a) a single molecular unit cell; b) the same as in $a$ ), but with the introduction of parcial disorder; c) an isotropic liquid and $d$ ) a molecular oriented liquid.

\section{NQR spectrum from $T_{1}(\nu)-$ profile dips}

\section{IIII QDs experimental procedure}

The phenomenon known as QDs is due to a resonant effect occurring when the Zeeman levels of a spin specie (protons ${ }^{1} H$ in our case) and the Zeeman-perturbed quadrupolar spin system $\left({ }^{14} N\right.$ in the present) match. Externally one can move the time scale -or more specifically the Larmor precessional frequency- of protons, until the two spin species can see in between them. Both spin species are coupled to the lattice; i.e, protons by dipolar magnetic interactions and nitrogens 
principally through electric interactions, and in addition both are externally compelled to couple between them through the dipole-dipole magnetic interaction. As compared with protons, quadrupolar nuclei are in general strongly coupled to the lattice and their spinlattice relaxation rate is thus much faster. If the levelcrossing condition has been reached by the experimental arrangement, a net flow of energy takes place from protons to quadrupolar nuclei and then to the lattice. The measurable consequence of this resonant process is an increment in the proton relaxation rate just at the Larmor frequencies of ${ }^{1} \mathrm{H}$ which are coincident with the ${ }^{14} \mathrm{~N}$-NQR resonances. Measuring the spin-lattice relaxation time $T_{1}$ of protons at Larmor frequencies scanned over a broad range, the ${ }^{14} N$ spectrum is thus recorded through the dips observed in the $T_{1}\left(\nu_{L}\right)$-profile. A comprehensive analysis of the ${ }^{14} \mathrm{~N}-{ }^{1} \mathrm{H}$ energy exchanges and involved relaxation processes at various ${ }^{1} H$-NMR and ${ }^{14} \mathrm{~N}$-NQR overlapping spectra situations, together with applications in some living systems, are given in refs. [15] and [16].

The experimental set-up is home made [17], the design is based in the Electronically Switched Fast Field Cycling NMR spectrometer, first developed at the Noack's Laboratory -Stuttgart- [18], which use a special air core magnet design [19] and a MOSFETs magnet control and power switching [20].

\section{IIIII First experimental results}

The firsts NQR studies in liquid crystals where carried out ten years ago $[10,11]$. Fig. 3 shows respectively the cases of $H p A B$ in the $\mathrm{SmC}$ and $\mathrm{N}$ mesophases at $\mathrm{T}=$ 78,82 and $110^{\circ} \mathrm{C}$. Extensions to other thermotropic liquid crystals, having only one ${ }^{14} \mathrm{~N}$ in its molecular structure, are shown in Fig. 4. In all the compounds and mesophases, the spectra are more similar to that of Fig. $2 \mathrm{~b}$ (dispersed anisotropic EFGs) than that of Fig. 2d (characteristics of "rod-like" LCs).

The nucleus of Chlorine presents a quadrupole moment approximately one order of magnitude higher than ${ }^{14} N$, therefore QDs can be expected in the tenth $\mathrm{MHz}$ regime. In order to check if QDs are a phenomenon itself or simply an effect on $T_{1}\left(\nu_{L}\right)$ produced by cycling the Zeeman field, studies involving ${ }^{1} \mathrm{H}$ and ${ }^{35} \mathrm{Cl}$ QDs were performed in a chlorinated LC by means of the conventional NMR technique, without the use of Field Cycling [12]. In addition, it was possible to separate the $T_{1}$-profile from the molecular chain and core protons. Dips on the $T_{1}\left(\nu_{L}\right)$ of protons neighboring the ${ }^{35} \mathrm{Cl}$ nucleus (belonging to the molecular core) are, or course, more deply (see Fig. 5 ).
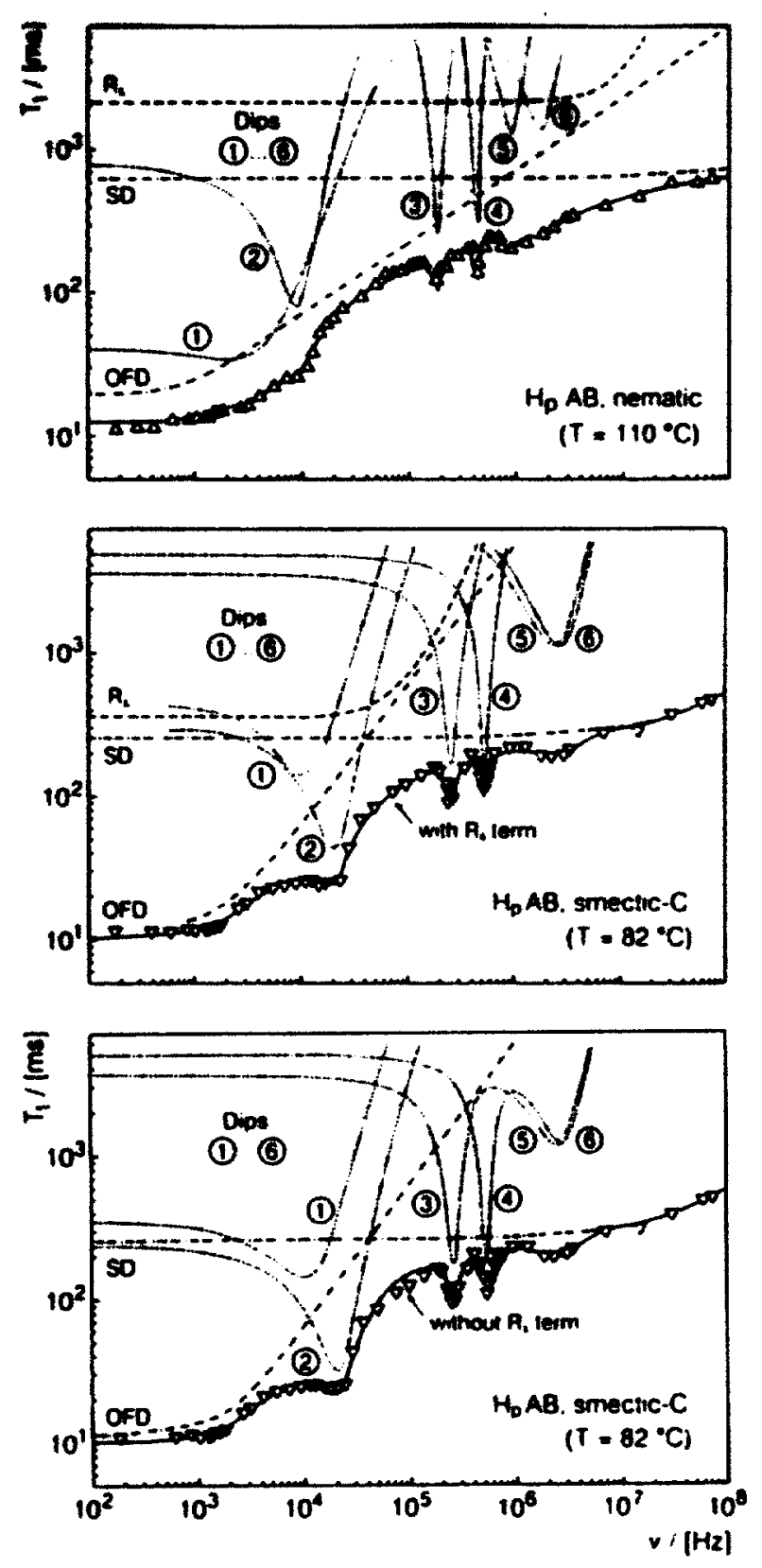

Figure 3. QDs obtained at the F. Noack's Laboratory, Stuttgart, in $H p A B$. A high Larmor frequencies unresolved doublet, followed to low frequencies by a nicely resolved pair of peacks and, finally, an unresolved very low frequencies doublet is observed in both mesophases [10]. The apparently two unequivalent nitrogens nuclei of the molecules sense different biaxial EFGs. 

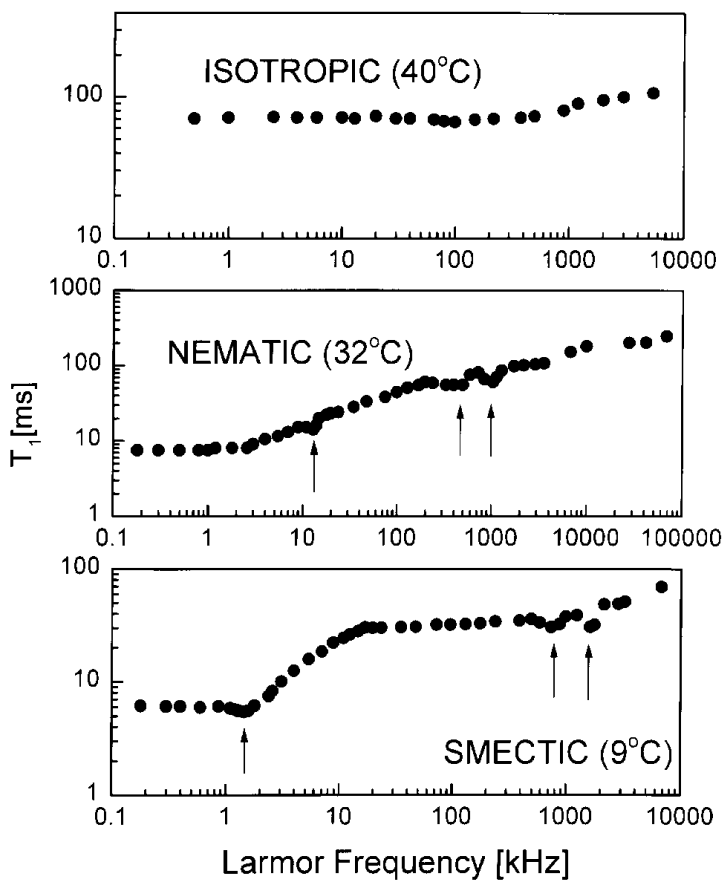

Figure 4. $T_{1}\left(\nu_{L}\right)$ profiles obtained in a LC with a single nitrogen atom in the molecule (8CB). A high frequency doublet and a singlete at the left of the relaxometry profile is observed in both mesophases. The three NQR lines indicate again biaxially EFG tensor at the single ${ }^{14} N$ site. See ref. [10] for details. QDs are absent in the isotropic phase.

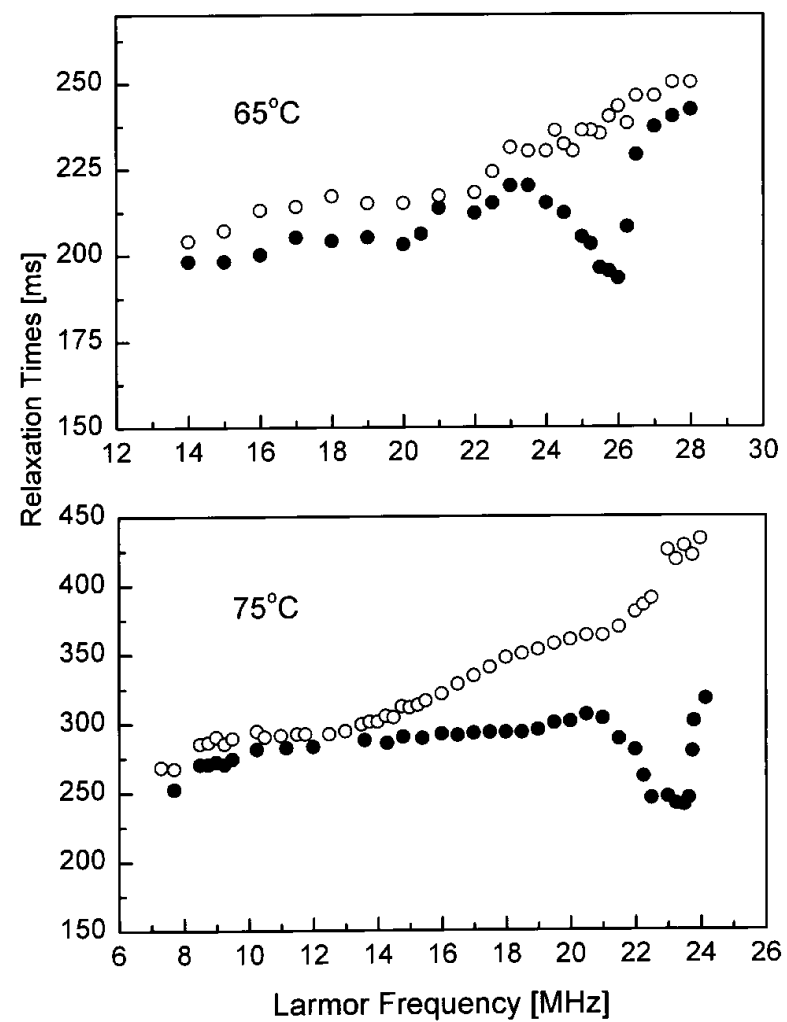

Figure 5. a) Experimental results of the spin-lattice relaxation times at $T=65^{\circ} \mathrm{C}$ and $T=75^{\circ} \mathrm{C}$. Empty circles represents the Larmor frequency behaviour of $T_{1}^{\text {alkyl }}$. Quadrupole dips are clearly distinguished in the $T_{1}^{a-c}$ behaviour (filled circles).
After that first studies, narrow QDs were measured by Dvinskikh and Molchanov [24] in ethoxybenzidenbutylanilin EBBA and by Zolino [21] in p-azoxyanizole PAA. More recently we worked out extensive refinements in the experimental technique in order to carefully control those parameters of the experiment which broad the spectral distribution of QDs: i.e., i) the stability and precision of the Zeeman field during the level crossing period; ii) the temperature gradients along the sample volume; and iii) the purity of the sample. See refs. [22] and [23] for details.

\section{QDs measurements in SmC- HpAB}

In Fig. 6 we depict the $T_{1}\left(\nu_{L}\right)$ of SmC- $H p A B$ at $\mathrm{T}$ $=82{ }^{\circ} \mathrm{C}$ measurements, within the spectral regions where QDs were detected. Nine $T_{1}\left(\nu_{L}\right)$ QDs have been found!: a low frequencies single dip at $\nu_{L} \approx 30 \mathrm{kHz}$; two high frequencies doublets at 500 and $800 \mathrm{kHz}$ and a higher frequencies quadruplet. This rich in peaks spectral structure can only be explained by assuming more than two chemically unequivalent nitrogen nuclei capable to contribute to the protons spin-lattice relaxation dispersion. Note that what we have done here was merely to resolve an internal structure in the $T_{1}\left(\nu_{L}\right)$ profiles previously studied [10]. At this altitude we can assign -within a certain degree of safety- the relaxometry spectrum to a bimolecular unit cell in the SmC$H p A B$ mesophase [22], where one of the nitrogens of a molecule is not equivalent with its pair of the partner one in the elemental unit cell. See Fig. 7 where we schematize the possible situation. There are two equivalent nuclei, one of each molecule, for instance $N_{2}$ and $N_{4}$. Following the molecular structure of $H p A B$, we can assume that the electronic charge of the oxygen atom provokes higher $\eta$ and $\kappa$ at the $N_{1}$ nuclear positions -one in each molecule-. We will discuss again the assignment of QDs together with pure NQR spectrum acquired with the double resonance technique. In this case we can conclude that three unequivalent nitrogens from the two molecules having biaxial EFGs can produce the observed nine QDs in the $T_{1}\left(\nu_{L}\right)$-profile.

What we believe is that in the $\mathrm{SmC}$ mesophase still alive certain degree of local molecular order from the solid crystalline state; i.e., $H p A B$ crystallize in monoclinic symmetry with two molecules per unit cell [25], and of course the NQR spectrum is rather complex; 
when the crystalline-SmC phase transition takes place, the inter-unit cell forces rather vanishes, but the thermal agitation is not enough to average the bonding force in between the two molecules of the unit cell.
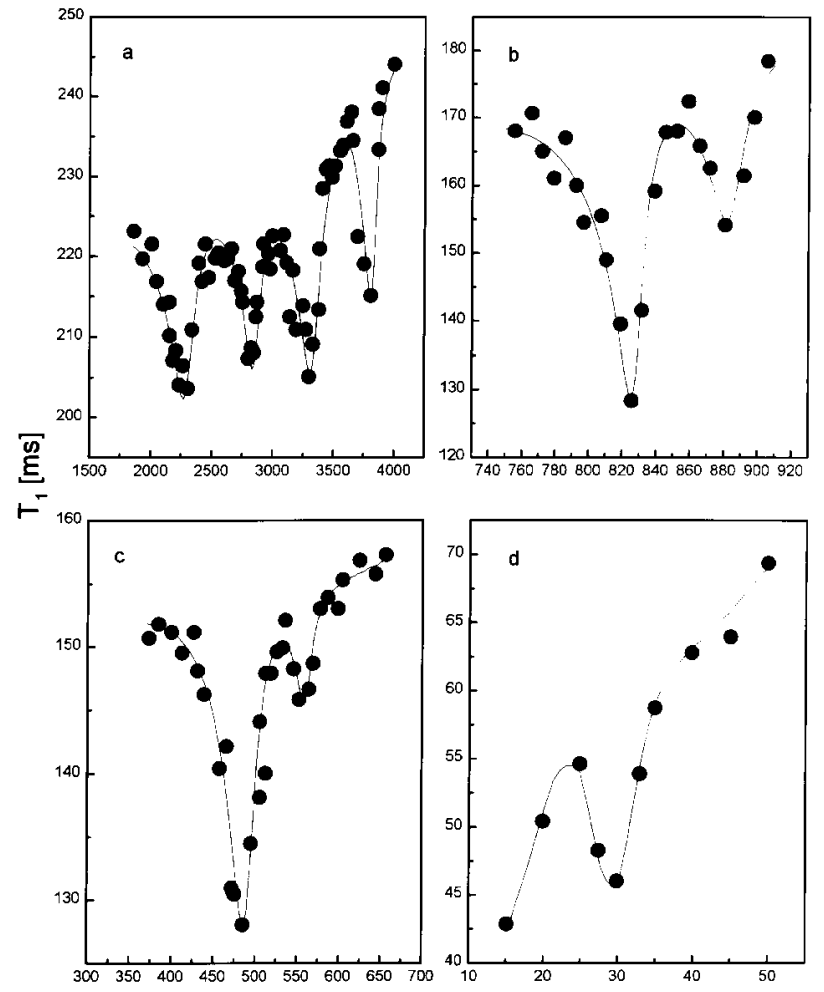

Larmor Frequency $[\mathrm{kHz}]$

Figure 6. Experimental QDs in the $T_{1}\left(\nu_{L}\right)$-profile, measured in $\mathrm{SmC}-H p A B$ at $\mathrm{T}=82{ }^{\circ} \mathrm{C}$. We can compute nine QDs in the $\nu_{L}$ range from $1 \mathrm{kHz}$ to $4 \mathrm{MHz}$. In the figure only the $T_{1}\left(\nu_{L}\right)$-profiles in the interes range are represented. Two unequivalent $H p A B$ molecules form the elementary microscopic units of the LC mesophase, see text.

For a description from a referential system mounted on the proper molecule, as the present is, the electric field gradients of every one of the four nitrogens involved in the LC-bimolecular unit cell are biaxial. We conclude these $T_{1}\left(\nu_{L}\right)$ profile studies classifying the SmC mesophase of $H p A B$ as a partially disordered molecular system.

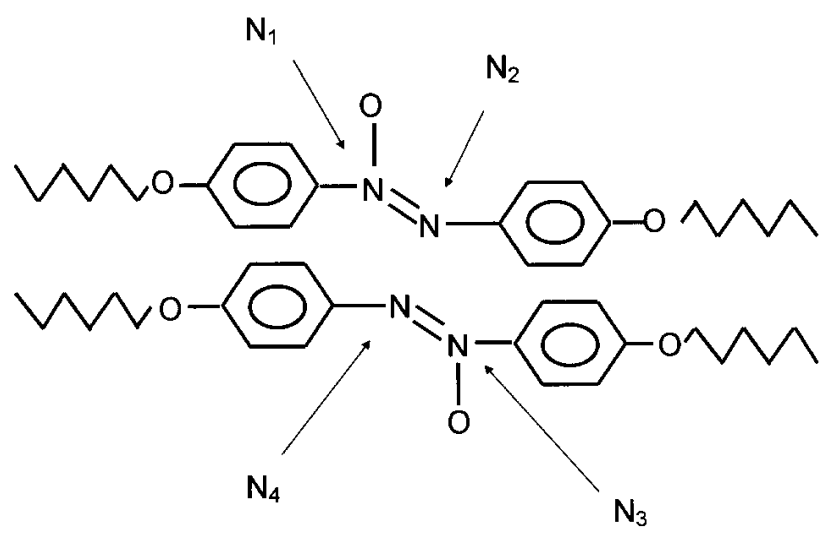

Figure 7. Schematic molecular arrangement proposed for the elementary unit cell of SmC-HpAB.

\section{Nuclear Quadrupole Double Resonance}

As in the case of the above described QDs in the relaxometry profile technique, the NMR of protons is used to evaluate the -in this case- zero Zeeman field NQR spectrum. It is currently referred as the "pure NQR spectrum". NQDOR combine electronically switched fast magnetic -or Zeeman- field cycling with two different radiofrequency excitations of the sample. The experiment can be summarily described as consisting in five different phases:

1. Polarization of protons in a high Zeeman field.

2. Adiabatic demagnetization of the sample, involving proton-nitrogen level crossing during the passage to zero magnetic field -at this stage the protons transfer magnetic polarization to nitrogens-.

3. Irradiation of nitrogens during the zero-magnetic field period, by means of a second radiofrequency coil perpendicular to the Zeeman field direction. If the second irradiation frequency $\nu_{Q}$ is in resonance with quadrupole nuclear transitions it can saturate the nitrogen quadrupole levels.

4. Switching on the Zeeman field, producing a new thermal contact between ${ }^{1} \mathrm{H}$ and ${ }^{14} \mathrm{Nby}$, again, level crossing.. If the second irradiation pulse was $" \frac{\pi}{2} "$, then ${ }^{14} \mathrm{Ns}$ will loss even more polarization.

5. Detection final step, evaluates the destruction of the ${ }^{1} \mathrm{H}$ magnetization during the cycle.

By scanning $\nu_{Q}$ step by step repeating the double resonance cycles, the effective NQR spectrum can be 
recorded by the evaluation of the NMR signal of protons during each detection stage.

At the time to design the experiment, some cares might be taken into account:

1. the irradiation zero-field period should be short enough in order to avoid ${ }^{1} H$-signal attenuation by $T_{1}$ relaxation.

2. the magnetic field switching on and off should be performed within the adiabatic demagnetization (and magnetization) condition [26].

The NQDOR spectrometer is a modified version of our home-made fast field cycling NMR relaxometer. The same rf coil is used for irradiation at both $\nu_{L}$ and $\nu_{Q}$. A reed relay bank connect that coil with the respective high and low frequency circuits.

\section{Experimental NQDOR in SmC and $\mathbf{N}$ mesophases of $H p A B$}

The $H p A B$ sample and thermal manipulation used for the NQDOR study are both the same than for QD$T_{1}$ relaxometry described above. Fig. 8 shows the NQDOR spectrum in the SmC- $H p A B$ at $\mathrm{T}=82{ }^{\circ} \mathrm{C}$. Both the frequency positions of the quadrupole resonances and the line widths are compatible with the dips in $T_{1}\left(\nu_{L}\right)$-profile shown in Fig. 6. Of course, small corrections on the QDs spectral positions due to the perturbing Zeeman field should be carried out for a right comparison. By technical reasons we avoid in this study the detection of the NQR transition at the lowest frequencies, but in any way the information that we could get from this resonance is redundant for the present study (see reference [18] for details). The NQDOR resonances at $800 \mathrm{kHz}$ and $837 \mathrm{kHz}$ are strong as compared with the others. we can then assign this doublet to the unresolved $\nu_{-}$and $\nu_{+}$of the equivalent nitrogens $N_{2}$ and $N_{4}$. The oxygen atom play a decisive role in the value of the quadrupolar coupling constant and in the respective EFG asymmetry parameters of the directly bounded nitrogens: $N_{1}$ and $N_{3}$ respectively. Instead $N_{2}$ and $N_{4}$ presents that small $\eta$ and $\kappa$, but we are not capable to separate $\eta_{2}$ from $\eta_{4}$ and $\kappa_{2}$ from $\kappa_{4}$.
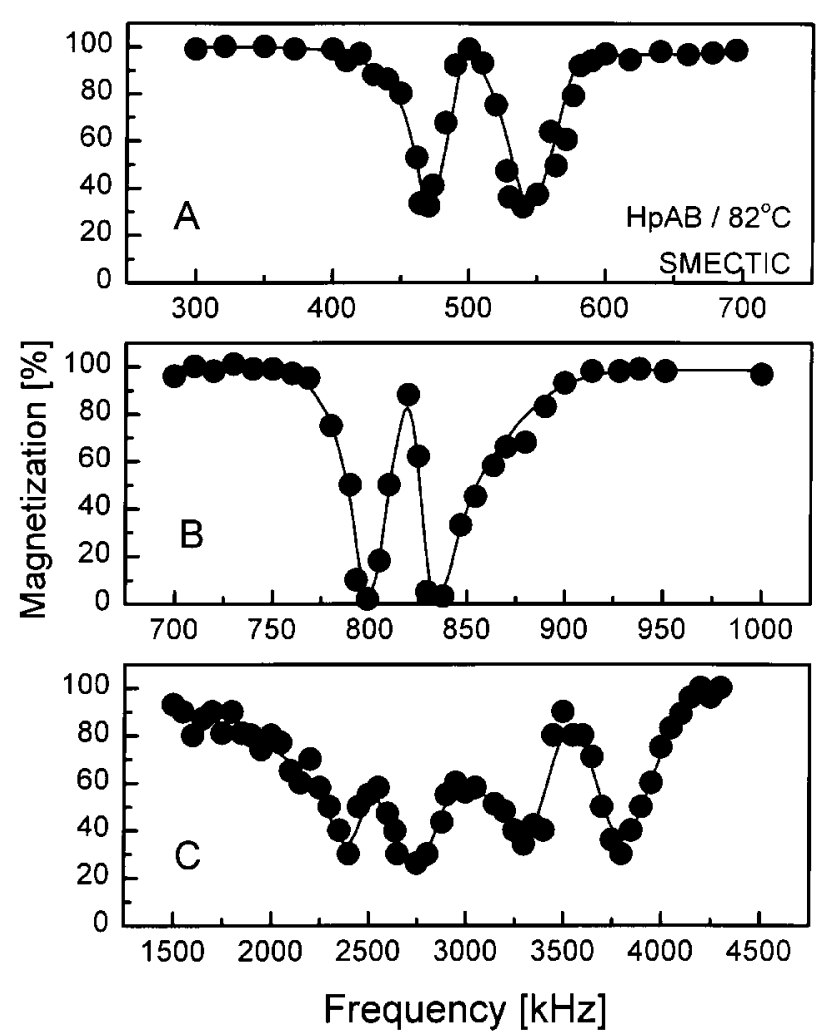

Figure 8. NQDOR spectra of $\mathrm{HpAB}$ at $\mathrm{T}=82{ }^{\circ} \mathrm{C} .{ }^{14} N$ resonances were found to be in close agreement with previous data of QDs found in the spin-lattice relaxation time experiment reported in ref. [10]. Solid line was built up by successive smoothing and FFT filtering of data just to guide the eye.
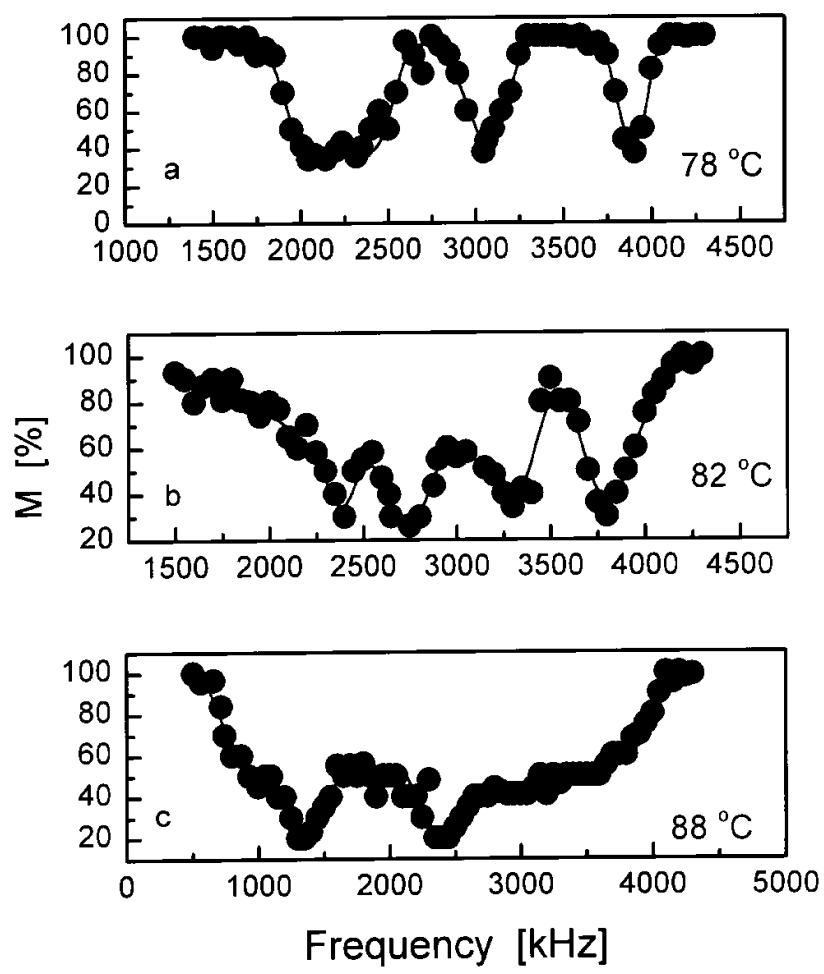

Figure 9. Temperature effect on the high frequency region of the NQDOR spectra for the Sm C mesophase of HpAB. 
In Fig. 9 we compare the high frequency quadruplet at three temperatures. Here the thermal effect is observed in the width of the NQR resonances. At low temperatures in the $\mathrm{SmC}$ mesophase $\left(T=78^{\circ} C\right)$, the NQR absorptions are relatively narrow and when we increase the temperatures approaching the $\mathrm{SmC}$ $\mathrm{N}$ phase transitions, the NQR spectrum tends to be broad (see Fig. 9). The behavior is not surprising because it is expected that thermally activated fluctuations increase the EFG dispersion. We can expeculate at this point that there should be a certain ratio of bi and uni-molecular unit cells and, as much as we approach the transitional temperature the quantity of singles molecules increase. That single molecules could rotate more or less freely following the long axis. Such molecules as described in Fig. 2d could contribute to the NQR spectrum with a single and extremely broad line, or in other words, with a broad distribution of resonance frequencies. That effect can explain the superimposed background and peaks structure at $\mathrm{T}=$ $88^{\circ} \mathrm{C}$ (Fig. 9c). Within that description we can propose the survival of two microscopic structures for the intermolecular EFG: uniaxial and biaxial. The biaxial EFGs provides the peaks in the NQR spectrum and the uniaxial (or EFG of ${ }^{14} \mathrm{~N}$ of oriented and rapidly rotating molecules) the background. The proportion of "uniaxial molecules" increases as the compound is heated and approaching to the SmC-N phase transition.

Even more rich are the studies on the nematic mesophase of HpAB. Figure 10 shows the NQR spectrum studied at the nematic phase at $\mathrm{T}=98{ }^{\circ} \mathrm{C}$, just $3{ }^{\circ} \mathrm{C}$ above the SmC-N transition. The present is, in our knowledgement, the first pure NQR experiment carried out in a nonsolid molecular system. Again, a complex peak structure is measured. We detect ten resonance lines; the unresolved doublet in now separated in a quadruplet. As compared with the SmC-NQR spectrum in the N-phase, the resonances shift to lower frequencies and tend to be broad. This result should not be surprising because the rotations following the short axis are expected to be more free in the nematic as compared with the $\mathrm{SmC}$ mesophase. Short axis rotations should of course average the EFG and then the coupling constants $\kappa$. Therefore, at this point we can conclude assuming microscopic biaxiality in the EFG at all the now four non equivalent ${ }^{14} N$ 's of the elemental unit cell in the nematic mesophase of $H p A B$. Even more we can propose that the number of molecules in the bimolecular unit cell is greater in the $\mathrm{N}$-mesophase at low temperatures as compared with the $\mathrm{SmC}$ phase at the temperature range close the $\mathrm{SmC}-\mathrm{N}$ phase transition; i.e., the molecules present a more ordered microstructure as much as the liquid crystal system cross from $\mathrm{SmC}$ to N mesophases. Finally, let's say that the nematic phase at low temperatures and from the microscopic point of view behaves as a partially disordered molecular system.

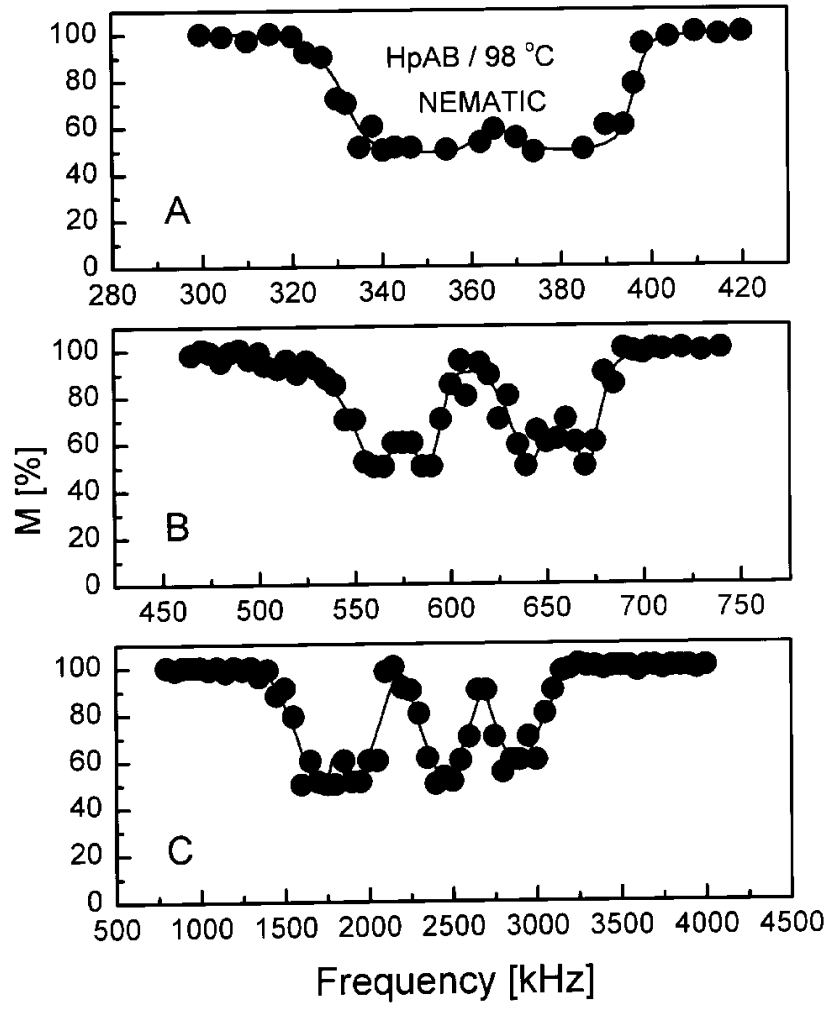

Figure 10. NQDOR spectra in the same experimental conditions but at $\mathrm{T}=98^{\circ} \mathrm{C}$ in the $\mathrm{N}$ liquid crystalline phase. The resonances clearly indicate a bimolecular local structure. Note that as compared with the spectrum of Fig. 8, the complete resonances set was shifted to lower frequencies; as expected, because of the highly molecular mobility in the nematic structures.

In Fig. 11 we depict the NQR spectrum acquired in the $\mathrm{N}$-mesophase of $\mathrm{HpAB}$ in the high temperature range $\left(\mathrm{T}=119^{\circ} \mathrm{C}\right)$. The NQR spectral structure is similar to that predicted for an oriented liquid in Fig. 2d. We can therefore propose that the liquid crystalline unit cells at this temperature in the $\mathrm{N}$-mesophase are composed by individual molecules; or, in other words, the EFG at the now indistinguishable nitrogens are uniaxials. That molecular microarrangement could be compatible with the concept of a partially ordered molecular system. In this situation we can assume that we are in presence of an uniaxial nematic microstructure. 


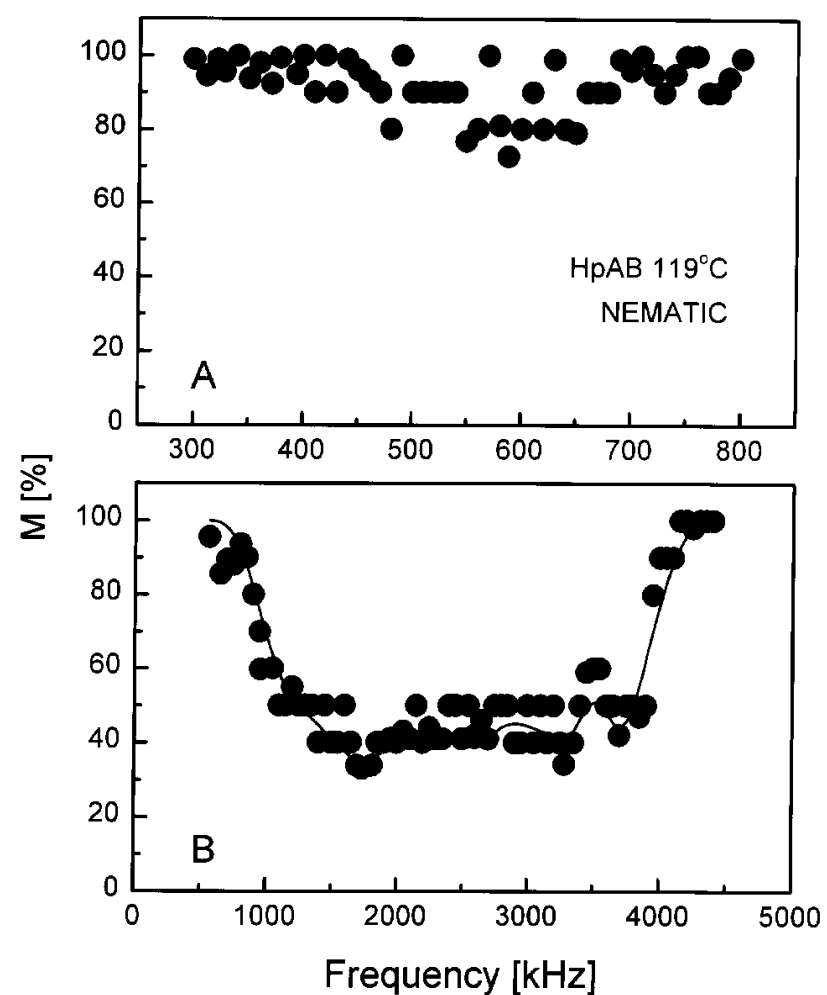

Figure 11. Experimental spectrum at high frequencies measured with the sample at $\mathrm{T}=119{ }^{\circ} \mathrm{C}$. A broad line replace the previous individual resonances spectrum (Fig. 10). An uniaxial local molecular organization including individual free rotations, is deduced from the single broad NQR line.

\section{Concluding Remarks}

In the sake of clarity we will divide conclusions in two fields of interest:

1. From the NQR-NMR techniques and applications:

- Quadrupolar Dips on $T_{1}\left(\nu_{L}\right)$ profiles can be observed in liquid crystals and very useful information on the molecular local microstructure can be deduced from them, but some cares on the experimental set up and methods should be considered.

- Our NQDOR experiments carried out in the liquid crystalline $\mathrm{HpAB}$ are also possible and should in principle be considered as the first performed in a non solid material.

2. From the liquid crystalline local microstructure:

- In the $\mathrm{SmC}$ mesophase of $\mathrm{HpAB}$ the local molecular arrangement presents a bimolecular elemental unit cell. A softening effect in the crystalline to smectic phase transition can be attributed to the inter-unit cell forces, while the two molecules composing the crystalline unit cell remain bonded in the whole $\mathrm{SmC}$ mesophase and even at low temperatures in the nematic mesophase.

- The bimolecular unit cell could coexist with some quantity of freely rotating individual molecules. As the $\mathrm{SmC}-\mathrm{N}$ transition is reached from bellow, the molecular fluctuations increase and consequently the quantity of "individual molecules" increase drastically.

- In the nematic mesophase and at low temperatures the majority of bimolecular unit cells is again recovered. The EFG of all the four nitrogens are biaxial. Of course, in other kind of description, where the reference system is fixed at the Laboratory -as it is the case of quadrupole perturbed NMRand/or if the time scale of the involved experiment is different to the present -infinite in optic or x-ray diffraction-, we could find thus uniaxial properties on the mesophase. From the microscopic point of view and in the condition of our experiments (time scale and referential system), we can think in this system as a partially disordered one.

- Finally, the transition to a local-partially ordered molecular system can be observed in the NQR - spectrum by increasing the temperature. The term "oriented liquid" can be interpreted as the thermal agitation is enough to broken the bimolecular microstructure and each molecule is now rotating individually and freely (once again within the micro-milliseconds time scale of our NQR experiment).

\section{Acknowledgments}

The authors thanks the Argentine public and private Science supporting Institutions (CONICET, CONICOR and Fundación Antorchas) and the German Alexander von Humboldt Foundation for financial support. One of us DJP thanks the crucial support of his wife Nora during the ten years of this work. This paper is dedicated to the memory of F. Noack. 


\section{References}

[1] H. Chihara and N. Nakamura, Adv. in Nuclear Quadrupole Resonance, vol. 4, Heydn, London (1981).

[2] D. Pusiol, A. E. Wolfenson and A. H. Brunetti, Phys. Rev. B 40, 2523 (1989).

[3] A. E. Wolfenson, D. J. Pusiol and A. H. Brunetti, Z. Naturforsch 45a, 334 (1990).

[4] A. E. Wolfenson, A. H. Brunetti, D. J. Pusiol y W. M. Pontuschka, Phys. Rev. B 41, 6257 (1990).

[5] S.R. Rabbani, N. Caticha, J.G. dos Santos y D.J. Pusiol, Phys. Rev. B 51, 8848 (1995).

[6] R. L. Armstrong and M. Ramia, J. Phys. C 18, 2877 (1985).

[7] M. Zuriaga and C. Martin, Z. Naturforsch 41a, 378 (1986).

[8] J. Seliger, R. Osredkar, V. Žagar and R. Blinc, Phys. Rev. Lett. 38, 411 (1977).

[9] R. Blinc, J. Dolinsek, M. Luzar and J. Seliger, Liq. Cryst. 3, 663 (1988).

[10] D. Pusiol and F. Noack, Liq. Cryst. 5, 377 (1989).

[11] D. Pusiol, R. Humpfer y F. Noack, Z. Naturforsch., 47a, 1105 (1992).

[12] E. Anoardo, D. Pusiol and C. Aguilera, Phys. Rev. B 49, 8600 (1994).

[13] D.T. Edmonds, Phys. Reports 29, 233 (1977).

[14] T.P. Das and E. Hahn, Nuclear Quadrupole Resonance Spectroscopy, Solid State Physics Series, Suppl. 1, Academic Press, New York (1958).
[15] R. Kimmich, F. Winter, W. Nusser and K. H. Spohn J. Mag, Res. 68, 263 (1986).

[16] R. Kimmich, NMR Tomography Diffusometry Relaxometry,

Springer Verlag; Berlin, Heidelberg, New York (1997).

[17] E. Anoardo, Thesis, Universidad Nacional de Córdoba (1996).

[18] F. Noack Prog. Nucl. Magn. Reson., 18, 161 (1986).

[19] E. Rommel, K. Mischker, G. Osswald, K.-H. Schweikaert and F. Noack, J. Magn. Reson. 70, 219 (1986).

[20] K.-H. Schweikaert, Thesis, Universität Stuttgart (1990).

[21] P. Zolino, Diplomarbeit, Universität Stuttgart (1994).

[22] E. Anoardo and D.J. Pusiol, Phys. Rev. Lett. 76, 3983 (1996).

[23] E. Anoardo and D.J. Pusiol, Phys. Rev. E 55, 7079 (1997).

[24] S. Dvinskikh and Y. Molchanov, Kimischeskaya Fiz., 10, 1204 (1994).

[25] J. Seliger, R. Osredkar, M. Mali and R. Blinc, J. Chem. Phys. 65, 2887 (1976).

[26] C.P. Slichter, Principles of Magnetic Resonance, Springer series in Solid-State Physics, 3rd Edition, Springer Verlag, Heidelberg (1990). 